\title{
Anticancer effects of isofraxidin against A549 human lung cancer cells via the EGFR signaling pathway
}

\author{
HAN ZHANG ${ }^{*}$, QIAN-QIAN FENG ${ }^{*}$, JIAN-HUA GONG and JING-PING MA \\ Breath Internal Medicine Department, The Second Clinical Medical College, \\ Jingzhou Central Hospital Affiliated to Tongji Medical College of Huazhong University of \\ Science and Technology and Yangtze University, Jingzhou, Hubei 434100, P.R. China
}

Received August 13, 2017; Accepted March 15, 2018

DOI: $10.3892 / \mathrm{mmr} .2018 .8950$

\begin{abstract}
Lung cancer is the leading cause of mortality due to tumor malignancy worldwide. In recent years, the treatment of lung cancer with chemotherapy has demonstrated notable resistance and insensitivity. Therefore, it is of great importance to investigate anti-lung cancer drugs with high efficiency and low toxicity. In the present study, the effects of isofraxidin on lung cancer cells and the associated mechanisms were investigated. The results revealed that, in vivo and in vitro, isofraxidin exhibited marked inhibitory effects on the A549lung cancer cell line. The results of Cell Counting kit-8, Transwell migration and Matrigel invasion assays, and flow cytometry to determine apoptosis, revealed that isofraxidin significantly inhibited the proliferation, migration and invasion of A549 cells, and induced the cell apoptosis. Furthermore, western blot analysis demonstrated that isofraxidin treatment led to effects on the expression of apoptosis-associated proteins, including members of the Bcl-2 protein family, and invasion-associated proteins, including matrix metallopeptidase (MMP)-2 and MMP-9, which may occur via inhibition of the expression of phosphorylated (p)-epidermal growth factor receptor, p-AKT and p-extracellular signal-regulated kinase. This regulation of protein expression may contribute to the inhibition of proliferation, migration and invasion of A549lung cancer cells by isofraxidin. In addition, despite the inhibitory effects on the A549 lung cancer cell line, the present study revealed that isofraxidin exhibited low toxicity towards BEAS-2B normal lung epithelial cells within a certain dose range $(0-160 \mu \mathrm{M})$,
\end{abstract}

Correspondence to: Dr Jian-Hua Gong or Dr Jing-Ping Ma, Breath Internal Medicine Department, The Second Clinical Medical College, Jingzhou Central Hospital Affiliated to Tongji Medical College of Huazhong University of Science and Technology and Yangtze University, 2 Renmin Road, Jingzhou, Hubei 434100, P.R. China

E-mail: gjh79911@163.com

E-mail: 2503398195@qq.com

*Contributed equally

Key words: isofraxidin, lung cancer, epidermal growth factor receptor signaling pathway, hypotoxicity, xenograft model indicating that isofraxidin may be employed for lung cancer treatment with hypotoxicity and fewer side effects. In conclusion, isofraxidin may be a novel candidate for anti-lung cancer chemotherapy.

\section{Introduction}

Lung canceris the leading cause of tumormalignancy-associated mortality worldwide. The morbidity of lung cancer has continuously increased and the overall five-year-survival rate is $\sim 15 \%$ (1), which poses a threat to public health.

Lung cancer, which is also termed lung carcinoma, may be divided into four major types: Small-cell lung carcinoma, adenocarcinoma, squamous cell carcinoma and large cell carcinoma. The latter three types are recognized collectively as non-small cell lung cancer, which accounts for $\sim 85 \%$ of all lung cancers $(2,3)$. At present, surgical operation is the most preferred and primary treatment for lung cancer at an early stage, which can completely remove the non-metastatic lesions to improve the long-term survival rate of patients $(4,5)$. However, the majority of lung cancers are detected at a late stage of tumor development, and in these cases the opportunity for surgery is lost and non-surgical treatments are relied upon, including chemotherapy, radiotherapy and targeted therapy. In recent years, non-surgical treatment of lung cancer has been associated with characteristics of chemotherapeutic toxicity, drug resistance and insensitivity $(6,7)$. Therefore, the development of novel anti-lung cancer drugs with high efficiency and low toxicity is essential.

Natural plants are an important source of novel pharmaceutical ingredients, and certain plants possess natural anticancer agents. Screening and investigating novel compounds from plants is an important strategy by which novel anticancer drugs with high efficiency and low toxicity may be identified (8). Isofraxidin is a coumarin compound that primarily exists in plants such as Sarcandraglabra and Acanthopanaxsenticosus (9). Isofraxidin has been reported to exhibit antibacterial, antioxidant, antidepressant and anti-inflammatory properties (10-12). Recently, isofraxidin was demonstrated to exert antitumor effects on two human colorectal adenocarcinoma cell lines, as the proliferation of HT-29 and SW-480 cells was inhibited by exposure to isofraxidin (9). Metastasis is the major factor associated with 
the recurrence and mortality of patients with lung cancer. Yamazaki and Tokiwa (13) indicated that isofraxidin may reduce the expression of matrix metallopeptidase 7 (MMP7) protein, thus inhibiting the invasive ability of liver cancer cells; however, to the best of our knowledge, the effects of isofraxidin on lung cancer and the associated mechanisms have not previously been reported. The present study investigated the effect of isofraxidin on the proliferation, apoptosis, migration and invasion of A549 lung cancer cells, as well as the underlying mechanisms, which provided theoretical and data support for the clinical application of isofraxidin in lung cancer.

\section{Materials and methods}

Reagents. Isofraxidin (Fig. 1A) was purchased from Shanghai Yuan Ye Biotechnology Co., Ltd. (Shanghai, China). Isofraxidin was dissolved in dimethyl sulfoxide (DMSO) as the stock solution, which was stored at $4^{\circ} \mathrm{C}$. The working solution of isofraxidin was diluted with Dulbecco's modified Eagle's medium (DMEM; Gibco; Thermo Fisher Scientific, Inc., Waltham, MA, USA) prior to use (the final DMSO concentration was $<0.5 \%$ in all cultures).

Cell lines and culture. A549 human lung cancer and BEAS-2B human normal lung epithelial cell lines were purchased from the Cell resource center of Shanghai institute of life sciences, Chinese academy of sciences (Shanghai, China). Cells were maintained in DMEM supplemented with $10 \%$ fetal bovine serum (FBS; Hyclone; GE Healthcare Life Sciences, Logan, UT, USA) and $100 \mathrm{U} / \mathrm{ml}$ penicillin and streptomycin in a humidified atmosphere containing $5 \% \mathrm{CO}_{2}$ at $37^{\circ} \mathrm{C}$. Cells were cultured to a confluency of $80 \%$ and were subsequently digested with $0.25 \%$ trypsin and seeded into new plates at the required density.

Cell counting kit-8 (CCK-8) assay. Cell proliferation was evaluated via a CCK-8 assay (Beyotime Institute of Biotechnology, Haimen, China). A549 and BEAS-2B cells were seeded in 96 -well plates at a concentration of $5 \times 10^{3} / 100 \mu 1$ per well and incubated overnight at $37^{\circ} \mathrm{C}$. The media were replaced with fresh media containing different concentrations of isofraxidin $(0-160 \mu \mathrm{M})$ and cells were cultured for 24,48 and $72 \mathrm{~h}$ at $37^{\circ} \mathrm{C}$ Subsequently, $10 \mu \mathrm{l} \mathrm{CCK}-8$ was added and cells were incubated for $4 \mathrm{~h}$ in the dark at $37^{\circ} \mathrm{C}$. The optical density (OD) at $450 \mathrm{~nm}$ was recorded by a microplate reader. The inhibitory rate (IR) of isofraxidin on cell proliferation was calculated using the following formula: IR $(\%)=\left(1-\mathrm{OD}_{\text {treatedgroup }} / \mathrm{OD}_{\text {control }}\right) \times 100$.

Cell cycle assay. Flow cytometric analysis was performed to determine the effects of isofraxidin on the cell cycle of A549 cells. A total of $4.5 \times 10^{5}$ A549 cells per $6 \mathrm{~cm}$ dish were exposed to isofraxidin $(0,10,20$ and $40 \mu \mathrm{M})$ at $37^{\circ} \mathrm{C}$ for $48 \mathrm{~h}$ and subsequently digested with EDTA-free trypsin. Cells were collected by centrifugation $\left(1,000 \times \mathrm{g}, 4^{\circ} \mathrm{C}, 5 \mathrm{~min}\right)$, fixed with cold $75 \%$ ethanol and incubated overnight at $4^{\circ} \mathrm{C}$. Cells were then stained with propidium iodide $[100 \mu \mathrm{g} / \mathrm{ml}$; Multi Sciences (Lianke) Biotech, Co., Ltd., Hangzhou, China] at $25^{\circ} \mathrm{C}$ for $30 \mathrm{~min}$ and flow cytometric analysis was performed immediately after staining (First-generation Attune flow cytometer,
Thermo Fisher Scientific, Inc.; Attune cytometric software, v2.1.0, Thermo Fisher Scientific, Inc.).

Hoechst 33342 staining. Hoechst 33342 (Beyotime Institute of Biotechnology, Shanghai, China) staining was used to detect the apoptosis of A549 cells treated with isofraxidin. A total of $1.5 \times 10^{5} \mathrm{~A} 549$ cells per well of 6 -well plate were exposed to isofraxidin $(0,10,20$ and $40 \mu \mathrm{M})$ at $37^{\circ} \mathrm{C}$ for $48 \mathrm{~h}$, followed by fixing with $4 \%$ formaldehyde for $20 \mathrm{~min}$ at room temperature. Cells were subsequently stained with Hoechst 33342 $(10 \mu \mathrm{g} / \mathrm{ml})$ at $25^{\circ} \mathrm{C}$ in the dark for $20 \mathrm{~min}$ and cell apoptosis was immediately evaluated with a fluorescent microscope (magnification, x100). Three fields of view were employed for analysis of apoptotic rate.

Migration and invasion assays. Transwell inserts (EMD Millipore, Billerica, MA, USA) were utilized for A549 cell migration and invasion assays. Cells were diluted with serum-free DMEM at the logarithmic growth phase and $5 \times 10^{4}$ cells in $200 \mu 1$ serum-free DMEM were added in each upper chamber. A total of $500 \mu 1$ DMEM medium containing $20 \%$ FBS was added into the lower chambers. In the invasion assay, Matrigel was also added to the upper well according manufacturer's protocol. After $1 \mathrm{~h}$ at $37^{\circ} \mathrm{C}$, isofraxidin was added to reach a concentration of $0,2.5,5$ and $10 \mu \mathrm{M}$. The concentrations of isofraxidin in upper and lower wells were in parallel. Following incubation at $37^{\circ} \mathrm{C}$ for $48 \mathrm{~h}$, the cells on the upper membrane were removed by cotton swab. The cells at the bottom of the membrane were fixed with $4 \%$ formaldehyde at $25^{\circ} \mathrm{C}$ for $20 \mathrm{~min}$, stained with $10 \%$ crystal violet at $25^{\circ} \mathrm{C}$ for 10-15 min and photographed under a light microscope (magnification, x200). Three fields of view were analyzed per sample.

Reverse transcription-quantitative polymerase chain reaction $(R T-q P C R)$. RT-qPCR was used to determine the transcript levels of the invasion-associated proteins MMP-2, MMP-9. A549 cells were exposed to isofraxidin $(0,10,20$ and $40 \mu \mathrm{M})$ for $48 \mathrm{~h}$ at $37^{\circ} \mathrm{C}$ and the total RNA of cells was extracted using TRIzol reagent (Thermo Fisher Scientific, Inc., Waltham, MA, USA). RT of isolated RNA was conducted (PrimeScript ${ }^{\mathrm{TM}}$ RT reagent kit; cat. no. RR037A, Takara Biotechnology Co., Ltd., Beijing, China) and amplified using the qPCR [ABI 7900HT Fast Real-Time PCR, (Applied Biosystems; Thermo Fisher Scientific, Inc.); SYBR-Green qPCR Master mix, cat. no. 638320; Takara Biotechnology Co., Ltd.]. Thermocycling conditions for qPCR: $95^{\circ} \mathrm{C}, 5 \mathrm{sec} ; 55^{\circ} \mathrm{C}, 20 \mathrm{sec} ; 72^{\circ} \mathrm{C}, 30 \mathrm{sec}$; 45 cycles. The $2^{-\Delta \Delta \mathrm{Cq}}$ method was employed for normalization. GAPDH was used as the control. Primer sequences used were as follows: MMP-2 forward, 5'-AGCGAGTGG ATGCCGCCTTTAA-3' and reverse, 5'-CAT TCCAGG CATCTGCGATGAG-3'; MMP-9 forward, 5'-GCCACTACT GTGCCTTTGAGTC-3' and reverse, 5'-CCCTCAGAGAAT CGCCAGTACT-3'; and GAPDH forward, 5'-GTCTCCTCT GACTTCAACAGCG-3' and reverse, 5'-ACCACCCTGTTG CTGTAGCCAA-3'.

Western blot analysis. A549 cells were treated with isofraxidin $(0,10,20$ and $40 \mu \mathrm{M})$ for $48 \mathrm{~h}$ at $37^{\circ} \mathrm{C}$ and collected. Cells lysis was conducted on ice for $30 \mathrm{~min}$ using lysis buffer, which consisted of a proteinase inhibitor mix, phosphatase inhibitor, 
A<smiles>COc1cc2ccc(=O)oc2c(OC)c1O</smiles>

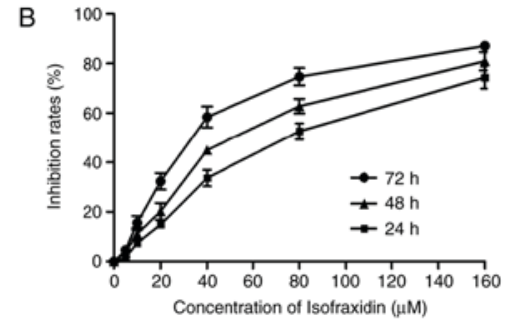

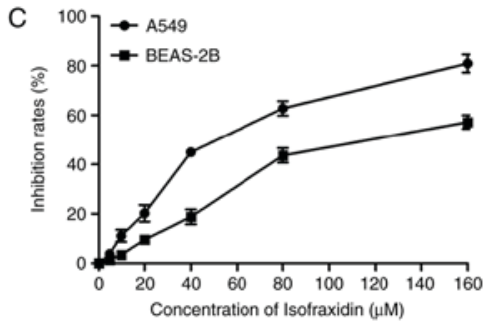

D

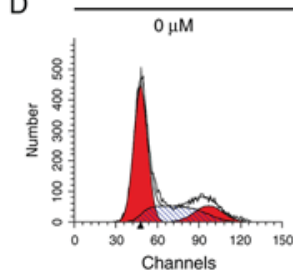

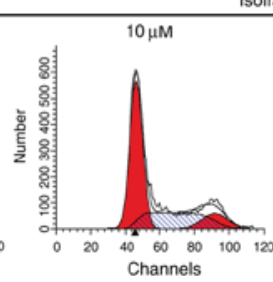

Isofraxidin
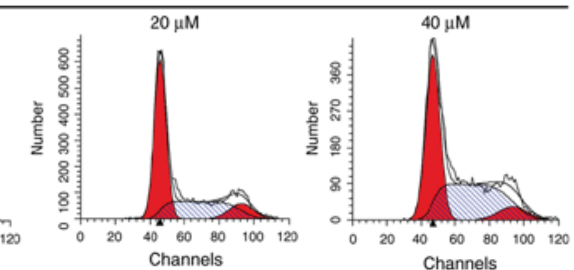

E

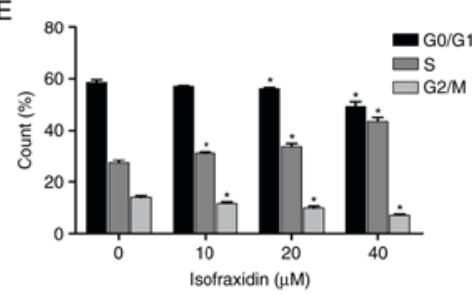

Figure 1. Isofraxidin inhibits the proliferation of A549 human non-small cell lung cancer cells. (A) Chemical structure of isofraxidin. (B) Cell proliferation of A549 cells exposed to isofraxidin at different concentrations for 24, 48 and $72 \mathrm{~h}$ was detected with a CCK- 8 kit. (C) Cell proliferation of A549 and BEAS-2B cells after $48 \mathrm{~h}$ treatment with isofraxidin at different concentrations was detected with a CCK-8 kit. (D) Representative flow cytometry graphs indicating cell cycle distribution of A549 cells following $48 \mathrm{~h}$ treatment with isofraxidin at different concentrations. (E) Percentage of A549 cells in each cell cycle stage after $48 \mathrm{~h}$ treatment with isofraxidin at different concentrations was calculated: $0 \mu \mathrm{M}(\mathrm{G} 0 / \mathrm{G} 1,58.63 \pm 0.94 \% ; \mathrm{G} 2 / \mathrm{M}, 13.97 \pm 0.74 \% ; \mathrm{S}, 27.38 \pm 1.14 \%), 20 \mu \mathrm{M}(\mathrm{G} 0 / \mathrm{G} 1$,

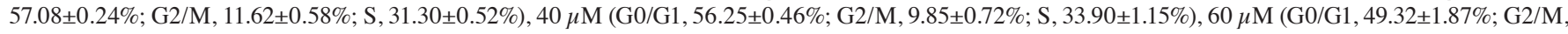
$7.08 \pm 0.39 \%$; $\mathrm{S}, 43.60 \pm 1.56 \%$ ). ${ }^{*} \mathrm{P}<0.05$ vs. $0 \mu \mathrm{M}$ isofraxidin. CCK- 8 , Cell Counting kit- 8 .

phenylmethylsulfonyl fluoride and radioimmunoprecipitation assay buffer (1:1:1:100; Beyotime institute of Biotechnology, Shanghai, China). The supernatant was collected following centrifugation, $10,000 \times \mathrm{g}, 30 \mathrm{~min}, 4^{\circ} \mathrm{C}$, the loading buffer was added and the mixture was boiled for $5 \mathrm{~min}$. The BCA method was used to determine the protein concentration and $10 \mu \mathrm{g}$ protein was loaded per loading well. Samples were analyzed by $10 \%$ SDS-PAGE and transferred electrophoretically to polyvinylidene difluoride (PVDF) membranes. The membranes were blocked with $10 \%$ skim milk solution at $25^{\circ} \mathrm{C}$ for $1 \mathrm{~h}$ and incubated overnight at $4^{\circ} \mathrm{C}$ with the following primary antibodies: Rabbit anti-epidermal growth factor receptor (EGFR) antibody (cat. no. 4267S), rabbit anti-phosphorylated (p)-EGFR (pY1068) antibody (cat. no. 3777S), mouse anti-AKT antibody (cat. no.2920S), mouse anti-p-AKT (pS473) antibody (cat.no.4060S), rabbit anti-extracellular signal-regulated kinase (ERK) antibody (cat. no. 4695S), rabbit anti-p-ERK (pT202/Y204) antibody (cat. no. 4370S), rabbit anti-Bcl-2-associated X (Bax) antibody (cat. no. 5023T), rabbit anti-Bcl-2 antibody (cat. no. 4223S), rabbit anti-MMP-9 antibody (cat. no. 13667T), rabbit anti-MMP2 antibody (cat. no. 40994S), rabbit anti-GAPDH antibody (HRP Conjugate; cat. no. 8884), which were purchased from Cell Signaling Technology, Inc., (Danvers, MA, USA). All primary antibodies were diluted in 1:1,000 proportion. GAPDH was used as the loading control. Following washing with TBST buffer (with $0.1 \%$ Tween-20), the PVDF membranes were incubated with species-specific horseradish peroxidase-conjugated secondary antibodies [anti-mouse IgG, horseradish peroxidase (HRP)-linked antibody; cat. no. 7076; anti-rabbit IgG, HRP-linked antibody; cat. no. 7074; Cell Signaling Technology, Inc., (Danvers, MA, USA), 1:5,000 dilution] at $25^{\circ} \mathrm{C}$ for $1 \mathrm{~h}$. The membranes were visualized using Pierce ${ }^{\mathrm{TM}}$ ECL Plus Western Blotting Substrate (Thermo Fisher Scientific, Inc.). Image Pro Plus 6.0 (Media Cybernetics, Inc., Rockville, MD, USA) was used for densitometry.
In vivoxenograft study. All procedures involving animals were approved by the Ethics Committee of the Jingzhou Central Hospital Affiliated to Tongji Medical College of Huazhong University of Science and Technology (Jingzhou, China). A total of 18 male BALB/c nude mice were purchased from the CAVENS Laboratory Animal Company (Changzhou, China; http://www.cavens.com.cn/). The average weight of the mice was $\sim 18 \mathrm{~g}$. They were maintained under specific pathogen-free conditions at $20-25^{\circ} \mathrm{C}$ (Humidity: 50-60\%; 12-h light dark cycle). A549 cells in PBS at $4 \times 10^{6} / 150 \mu 1$ per mouse were injected subcutaneously into the 4 -week-old nude mice. The mice were returned to their cages for continuous feeding. Ad libitum food and water was available. The tumor formation was regularly analyzed. The visible subcutaneous tumor was inspected by the naked eye. The tumor-bearing mice were separated into three groups: Negative control group ( $\mathrm{n}=6$ mice), isofraxidin-treated group $(5 \mathrm{mg} / \mathrm{kg}, \mathrm{n}=6 \mathrm{mice})$ and cisplatin-treated (Shanghai Yuanyeshengwu, Shanghai, China) positive control group ( $5 \mathrm{mg} / \mathrm{kg}, \mathrm{n}=6$ mice). Drugs were delivered daily by intraperitoneal injection for 21 days. All mice were sacrificed following the treatment period and the body weights and tumor volumes were measured. The expression levels of p-EGFR, p-AKT and p-ERK were detected by immunohistochemical staining as described previously (14).

Statistical analysis. All the assays were repeated in triplicate, data were processed using SPSS 17.0 statistical software (SPSS, Inc., Chicago, IL, USA) and the results are presented as the mean \pm standard deviation. The quantitative experiments were analyzed using analysis of variance. Dunnett's test was used as a post-hoc test. Statistical graphs were produced using GraphPad Prism 5 software (GraphPad Software, Inc., La Jolla, CA, USA). P<0.05 was considered to indicate a statistically significant difference. 


\section{Results}

Isofraxidin inhibits the proliferation of the A549 cell line. To determine the effect of isofraxidin on the proliferation of A549 cells and the optimal dosage, the cells were treated with isofraxidin at concentrations of $0,5,10,20,40,80$ and $160 \mu \mathrm{M}$ for 24,48 and $72 \mathrm{~h}$. The results revealed that isofraxidin exhibited a dose- and time-dependent inhibitory effect on the proliferation of A549 cells (Fig. 1B), with half-maximal inhibitory concentration $\left(\mathrm{IC}_{50}\right)$ values of $28.76 \pm 3.16$, $42.71 \pm 4.05$ and $75.16 \pm 3.42 \mu \mathrm{M}$ following 72,48 and $24 \mathrm{~h}$ of treatment, respectively. Furthermore, a lower rate of inhibition was observed in BEAS-2B normal lung epithelial cells following isofraxidin treatment compared with A549 cells (Fig. 1C), with an $\mathrm{IC}_{50}$ value of $85.32 \pm 2.34 \mu \mathrm{M}$ after $48 \mathrm{~h}$ of treatment, indicating that within a certain dosage range, isofraxidin exhibited a lower toxicity towards normal lung epithelial cells compared with cancerous cells. Additionally, alterations in the cell cycle were analyzed by flow cytometry, the results of which demonstrated that following treatment with isofraxidin for $48 \mathrm{~h}$, the cell cycle of A549 cells was markedly altered, particularly at concentrations of 20 and $40 \mu \mathrm{M}$, compared with the control cells (Fig. 1D and E). The percentage of cells in $\mathrm{S}$ phase increased in a dose-dependent manner (Fig. 1D and E), indicating that isofraxidin inhibited the proliferation of A549 cells by arresting cells in $\mathrm{S}$ phase.

Isofraxidin induces apoptosis in A549 cells in a dose-dependent manner. Drug-induced apoptosis was microscopically observed by Hoechst 33342 staining. A549 cells exposed to isofraxidin $(0,10,20$ and $40 \mu \mathrm{M})$ for $48 \mathrm{~h}$ were fixed, stained and observed under a microscope. The results demonstrated that the nuclei of cells in the control group were uniformly stained and the fluorescence intensity was not as strong compared with cells treated with isofraxidin. Increased fluorescence intensity in isofraxidin-treated cells appeared to be dose-dependent (Fig. 2A). When treated with $40 \mu \mathrm{M}$ isofraxidin, nuclear pyknosis and crescent-shaped nuclei were observed, indicating that isofraxidin may induce the apoptosis of A549 cells in a dose-dependent manner (Fig. 2A). To determine the expression of two apoptosis-associated proteins, Bcl-2 and Bax, western blot analysis was performed. The ill-defined band of lower molecular weight is assumed to be nonspecific. The results revealed that the expression of Bax was significantly upregulated, while the expression Bcl-2 was significantly downregulated, in response to isofraxidin treatment in a dose-dependent manner (Fig. 2B). This result further indicated that isofraxidin may induce the apoptosis of A549 cells.

Isofraxidin suppresses the migration and invasion of A549 cells. Metastasis is closely associated with the prognosis and recurrence of cancer $(15,16)$. To confirm the effects of isofraxidin on cell motility, Transwell migration and invasion assays were conducted. A549 cells were treated with isofraxidin at relatively low concentrations $(0,2.5$, 5 and $10 \mu \mathrm{M}$ ) for $48 \mathrm{~h}$, low concentrations of isofraxidin have little inhibitory effect on cell proliferation, which may affect the cell migration and invasion. The results revealed that the increase of isofraxidin concentration led to a decreased number of migratory/invasive cells, indicating that the migration and invasion of A549 cells was significantly inhibited compared with the corresponding controls (Fig. 3A). Western blot and RT-qPCR analyses of the expression of two invasion-associated genes, MMP-2 and MMP-9, revealed that the expression levels of MMP-2 and MMP-9 were significantly reduced following treatment with isofraxidin compared with control cells (Fig. 3B and C), which further confirmed the inhibitory effects of isofraxidin on the invasion of A549 cells.

Isofraxidin inhibits A549 cell proliferation, migration and invasion by inhibiting EGFR phosphorylation. Phosphoinositide 3-kinase (PI3K)/AKT and mitogen-activated protein kinase (MAPK)/ERK signaling pathways are important in the proliferation, apoptosis, invasion and migration of tumor cells (17-19). The alterations in p-AKT and p-ERK expression levels in A549 cells following isofraxidin treatment were detected by western blotting. The results demonstrated that the expression levels of p-AKT and p-ERK following treatment with isofraxidin was significantly inhibited in a dose-dependent manner (Fig. 4A and B). PI3K/AKT and MAPK/ERK signaling pathways are primarily regulated by receptor tyrosine kinases (RTKs) and EGFR is the principal member of the RTKs. Western blot analysis revealed that following treatment with isofraxidin, the expression levels of p-EGFR were significantly downregulated (Fig. 4C). Therefore, isofraxidin may inhibit the PI3K/AKT and MAPK/ERK signaling pathways by reducing the levels p-EGFR to hinder the development of lung cancer cells.

Isofraxidinsuppresses tumor growth in vivo. To determine whether isofraxidin has potential therapeutic value for the treatment of lung carcinoma, the effects of isofraxidin on A549 cells were investigated in vivo in the present study using a xenograft mouse model. The weights of tumor-bearing nude mice are presented in Fig. 5A. Compared with in the positive control group, isofraxidin treatment reduced the body weight of nude mice less, with body weights more similar to those in the negative control group, indicating the potentially low toxicity and side effects of isofraxidin. The volume of the xenografts of samples collected after 21 days of treatment via intraperitoneal injection are presented in Fig. 5B. Compared with in the negative control group, isofraxidin significantly reduced the volume of the xenografts. Furthermore, Immunohistochemical detection demonstrated that following treatment with isofraxidin, the expression levels of p-EGFR, p-AKT and p-ERK were significantly downregulated compared with in the control group (Fig. 5C), which was consistent with the aforementioned in vitro results of the current study. These data indicated that isofraxidin suppressed the growth of xenografts in mice.

\section{Discussion}

Natural compounds have frequently been used to treat cancers. At present, there are $>3,000$ anticancer drugs that are derived from plants, including vinblastine, vincristine, etoposide, paclitaxel and camptothecin (8). Isofraxidin is a 

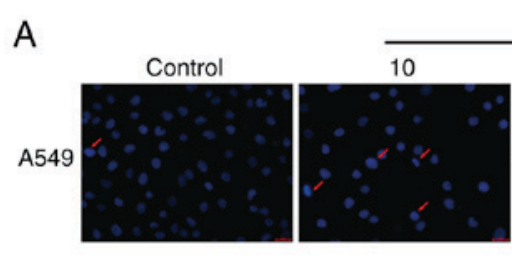

Isofraxidin $(\mu \mathrm{M})$
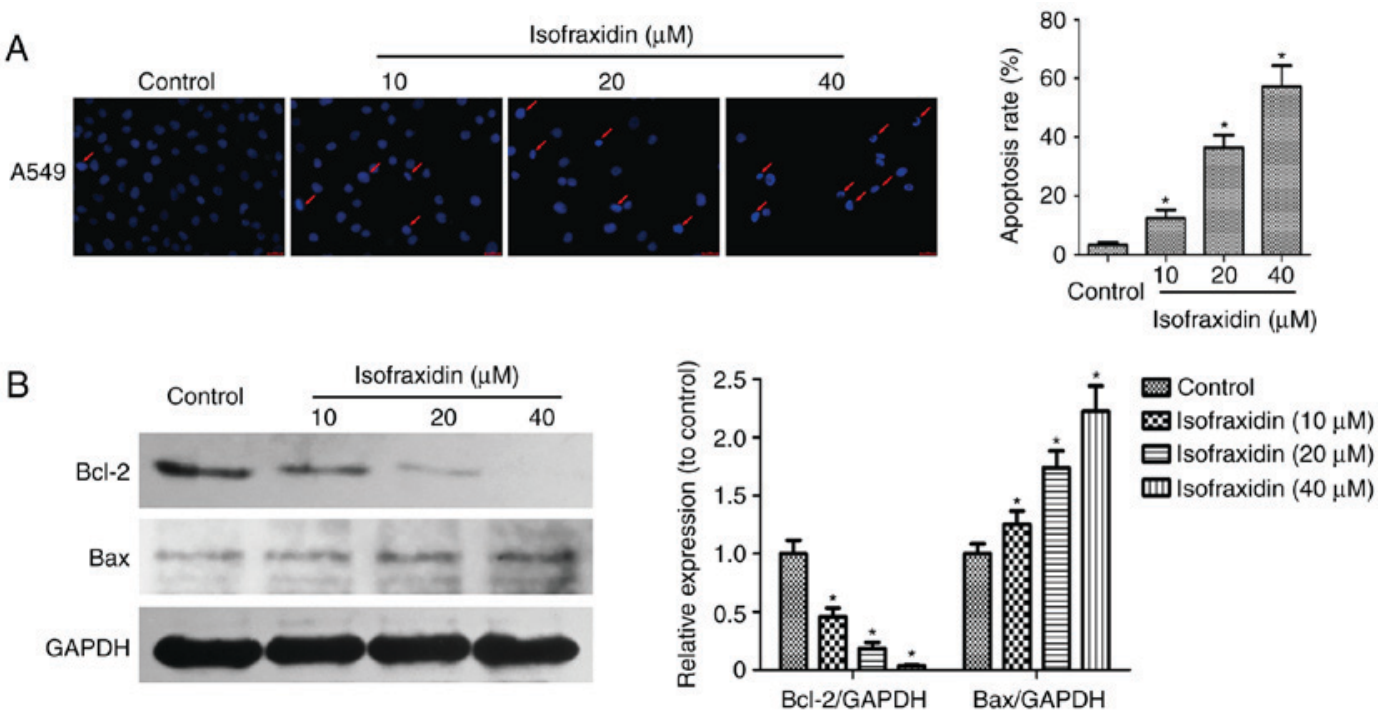

Figure 2. Isofraxidin induces A549 cell apoptosis. (A) Detection of A549 cell apoptosis. Arrows indicate the apoptotic cells. The histogram presents the apoptotic rate in each group. A549 cells were treated with isofraxidin for $48 \mathrm{~h}$ at different concentrations and stained with Hoechst 33342 (magnification, x400). Scale bar, $20 \mu \mathrm{m}$. (B) Western blot analysis of Bcl-2 and Bax protein expression levels after $48 \mathrm{~h}$ treatment with isofraxidin at different concentrations. The histogram presents semi-quantitative analysis of the results of western blotting. GAPDH was used as the loading control. "P<0.05 vs. control. Bax, Bcl-2-associated X.
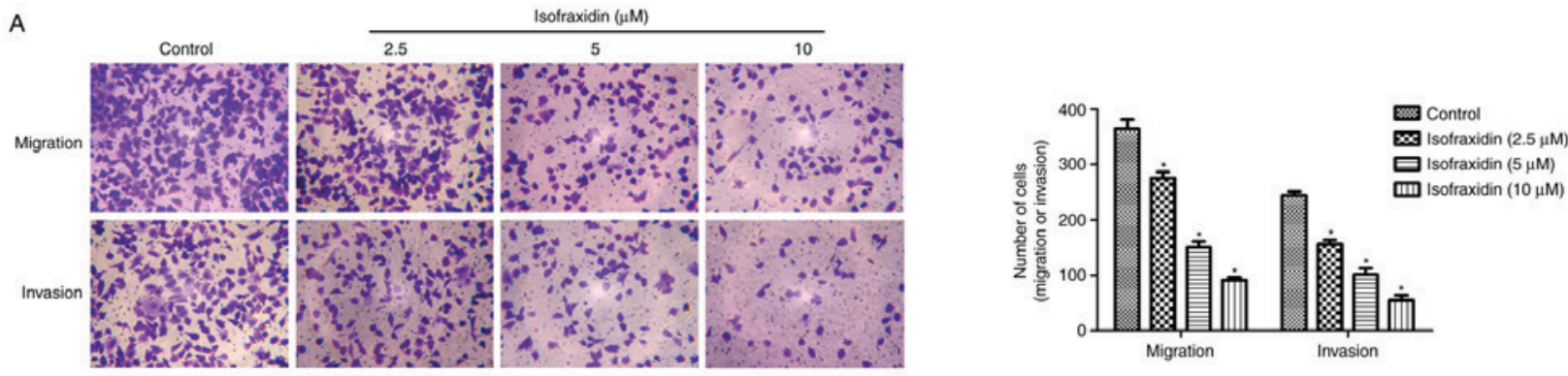

B
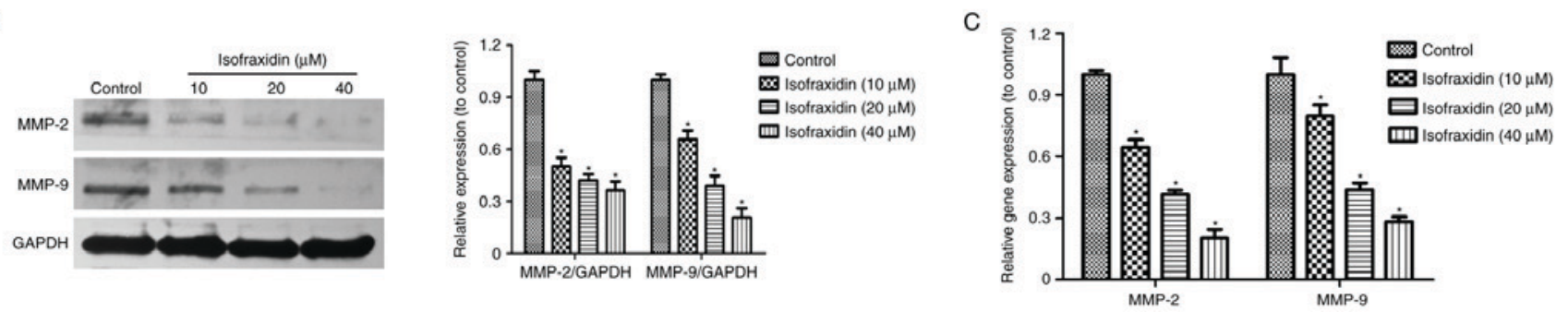

Figure 3. Isofraxidin inhibits the migration and invasion of A549 cells. (A) Transwell migration and Matrigel invasion assay results for the inhibitory effects of different concentrations of isofraxidin on the migration and invasion of A549 cells. Magnification, x200. (B) Western blot analysis of the expression levels of invasion-associated proteins, MMP-2 and MMP-9, after $48 \mathrm{~h}$ treatment with isofraxidin at different concentrations. The histogram presents semi-quantitative analysis of the results of western blotting. GAPDH was used as the loading control. (C) mRNA expression levels of MMP-2 and MMP-9 after $48 \mathrm{~h}$ treatment with isofraxidin at different concentrations were detected by reverse transcription-quantitative polymerase chain reaction. ${ }^{*} \mathrm{P}<0.05$ vs. control. MMP, matrix metallopeptidase.

coumarin compound that primarily exists inceratin plants, including Sarcandraglabra and Acanthopanaxsenticosus (9). Antibacterial, antioxidant, antidepressant, anti-inflammation and antitumor effects of isofraxidin have previously been reported (20). As one of the most common types of malignant tumor, lung cancer is associated with certain characteristics, including rapid development and high metastasis, which is associated with high mortality. To the best of our knowledge, the effect of isofraxidin on lung cancer has not previously been reported; however, the present study revealed for the first time that isofraxidin may inhibit the proliferation, migration and invasion of A549 cells, and induce cell apoptosis, by inhibiting the EGFR signaling pathway.

The proliferation, apoptosis, migration and invasion of tumor cells involves a series of signaling pathways, making it a complex process with particular mechanisms that require further investigation. Bcl-2 family proteins are the most important regulatory factors in the mitochondrial apoptosis pathway and are made up of two primary groups: Apoptosis-inducing (apoptotic) proteins, including Bax, Bcl-2 antagonist/killer 1 and peptidyl-tRNA hydrolase 2, andapoptosis-inhibiting (antiapoptotic) proteins, including 
A
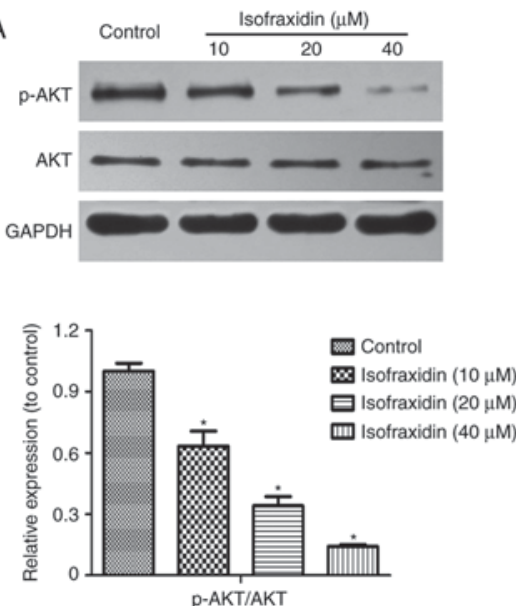
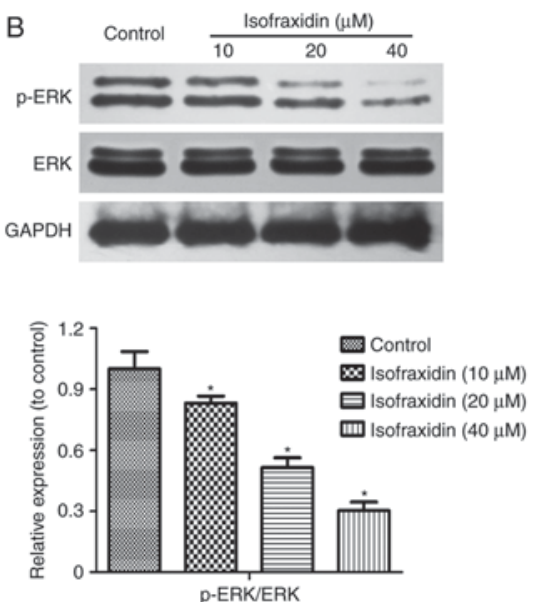
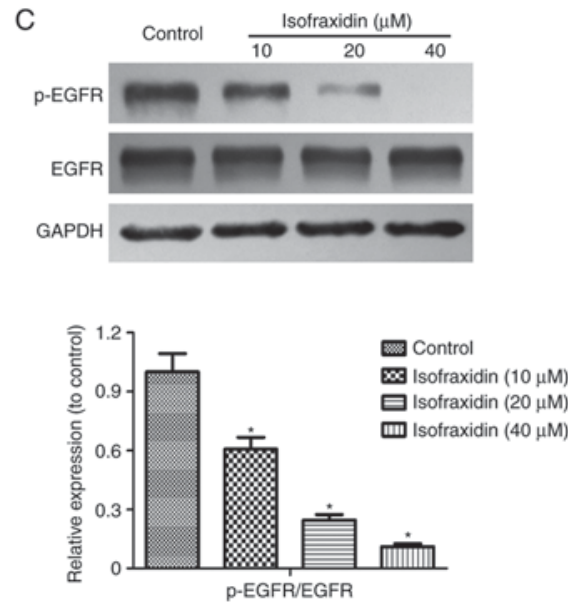

Figure 4. Isofraxidin inhibits the proliferation, migration and invasion of A549 cells by inhibiting the phosphorylation of EGFR. Western blot analysis of the expression levels of (A) p-AKT, (B) p-ERK and (C) p-EGFR after $48 \mathrm{~h}$ treatment with isofraxidin at different concentrations. GAPDH was used as the loading control. " $\mathrm{P}<0.05$ vs. control. The histograms present semi-quantitative analysis results of western blotting results. EGFR, epidermal growth factor receptor; $\mathrm{p}$-, phosphorylated-; ERK, extracellular signal-regulated kinase.

A
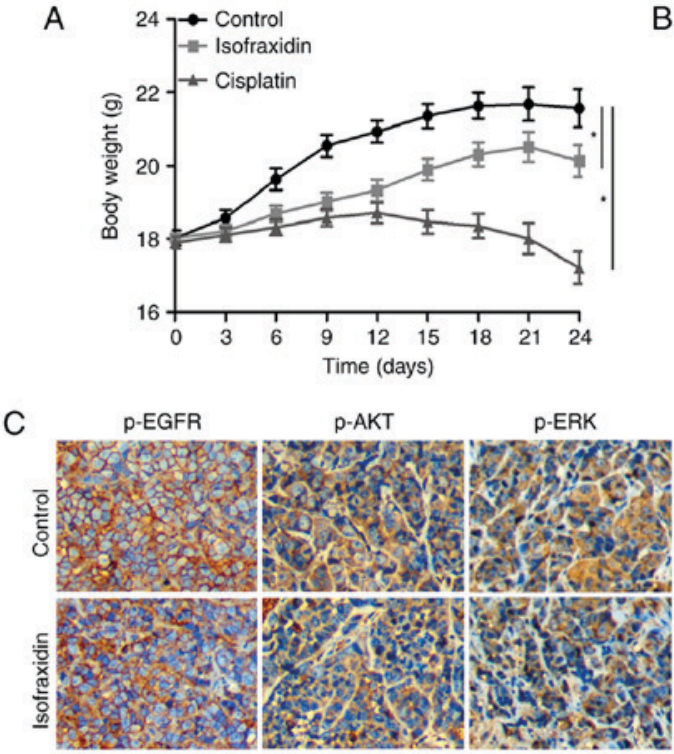
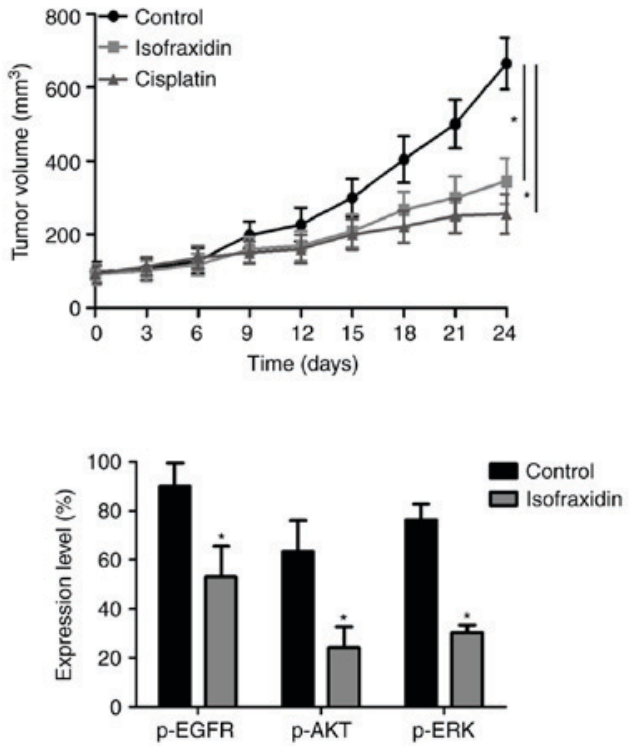

Figure 5. Isofraxidin inhibits the growth of lung cancer xenografts in mice. A lung cancer xenograft model was established by subcutaneous injection of A549 cells into nude mice. When the volume of the tumor was $\sim 90 \mathrm{~mm}^{3}$, isofraxidin and cisplatin were injected into mice via intraperitoneal injection for 21 days. Mice (A) body weight and (B) tumor volume were regularly monitored during treatment. (C) Expression of p-AKT, p-ERK and p-EGFR was detected by immunohistochemical analysis (magnification, $\mathrm{x} 400$ ). "P<0.05 vs. control or as indicated. p-, phosphorylated-; ERK, extracellular signal-regulated kinase; EGFR, epidermal growth factor receptor.

Bcl-2 and Bcl-2-like 1 (21-23). It was previously reported that isofraxidin significantly reduced the expression of the antiapoptotic protein $\mathrm{Bcl}-2$ in colon cancer cells and increased the expression of the apoptotic proteinscaspase-3, caspase-9 and Bax (9). Therefore, the present study detected the effect of isofraxidin on the expression of Bcl-2 protein family members. The results revealed that isofraxidin promoted the expression of Bax but reduced the expression of $\mathrm{Bcl}-2$ in a dose-dependent manner, indicating that isofraxidin may induce the apoptosis of A549 cells.

Patients with cancer succumb to the disease primarily due to the recurrence of malignant tumors and metastasis, and the key process resulting in the invasion and metastasis of malignant tumors is the degradation of the extracellular matrix (ECM).
MMPs are the major enzymes that degrade the components of the ECM (24-26). In the process of developing safe and effective anticancer drugs, analysis of the antimetastatic ability of the drug is particularly important; the inhibition of MMP activity is also regarded as one aim of cancer treatment. It has been demonstrated that isofraxidin may suppress the invasion of hepatocellular carcinoma by inhibiting the expression of MMPs (13). Therefore, the present study investigated the effect of isofraxidin on expression of the MMP family proteins and the results revealed that the expression levels of MMP-2 and MMP-9 were significantly reduced following treatment with isofraxidin. The results of the present study indicated that isofraxidin was able to suppress the migration and invasion of A549 cells, as confirmed by Transwell migration and invasion assays. 
PI3K/AKT and MAPK/ERK signaling pathways are important in the proliferation, apoptosis, migration and invasion of tumor cells. Numerous studies have reported that AKT may affect cell proliferation and apoptosis by regulating the Bcl-2 family-associated proteins $(27,28)$. The results of the present study revealed that isofraxidin reduced the expression of p-AKT in A549 cells; therefore, isofraxidin may affect the expression of the $\mathrm{Bcl}-2$ protein family by inhibiting the AKT signaling pathway, leading to the subsequent inhibition of the proliferation of A549 cells and the induction of apoptosis. ERK has been reported to regulate the expression of MMP family-associated proteins, thus affecting cell migration and invasion $(29,30)$. The present study also revealed that isofraxidin reduced the expression of p-ERK in A549 cells, indicating that isofraxidin may inhibit the expression of MMP-2 and MMP-9 proteins by inhibiting the ERK signaling pathway, subsequently inhibiting the migration and invasion of A549 cells.

RTKs are glycoproteins that span the cell membrane and bind a growth factor within its surface, which controls the transduction of cellular signals (31). There are numerous members of the RTK family, including EGFR, fibroblast growth factor receptor, vascular endothelial growth factor receptor and platelet-derived growth factor receptor (32). EGFR is considered to be among the most important members of the RTK family and its importance has been demonstrated by the clinical application of EGFR-targeting drugs, including gefitinib and afatinib (33).

Based on the effect of isofraxidin on PI3K/AKT and MAPK/ERK signaling pathways in the present study, isofraxidin may affect the activity of EGFR in lung cancer cells. The results of the present study revealed that the phosphorylation level of EGFR in lung cancer cells was significantly inhibited following treatment with treatment isofraxidin.

In conclusion, the present study confirmed the inhibitory effect of isofraxidin on lung cancer development. The results indicated that isofraxidin may inhibit the development of lung cancer cells by inhibiting the phosphorylation of EGFR and inhibiting the PI3K/AKT and MAPK/ERK signaling pathways. Furthermore, BEAS-2B human normal lung epithelial cells exhibited low sensitivity to isofraxidin within a certain dose range, indicating the potentially low toxicity and side effects of isofraxidin in the treatment of lung cancer. These results indicate the potential of isofraxidin as a novel candidate for anti-lung cancer chemotherapy.

\section{Acknowledgements}

Not applicable.

\section{Funding}

No funding was received.

\section{Availability of data and materials}

All data and materials described in this manuscript are available from the correspondence author upon reasonable request.

\section{Authors' contributions}

$\mathrm{HZ}$ and QQF performed the experiments. JHG and JPM analyzed the data and wrote the manuscript.

\section{Ethics approval and consent to participate}

All procedures involving animals were approved by the Ethics Committee of the Jingzhou Central Hospital Affiliated to Tongji Medical College of Huazhong University of Science and Technology (Jingzhou, China).

\section{Consent for publication}

Not applicable.

\section{Competing interests}

The authors declare that they have no competing interests.

\section{References}

1. Chang A: Chemotherapy, chemoresistance and the changing treatment landscape for NSCLC. Lung Cancer 71: 3-10, 2011.

2. Lamb YN and Scott LJ: Osimertinib: A review in T790M-positive advanced non-small cell lung cancer. Target Oncol 12: 555-562, 2017.

3. Goss GD and Spaans JN: Epidermal growth factor receptor inhibition in the management of squamous cell carcinoma of the lung. Oncologist 21: 205-213, 2016.

4. Salmenkivi K and Knuuttila A: Diagnostics of non-small cell lung carcinoma. Duodecim 130: 701-704, 2014 (In Finnish).

5. Rosenzweig K: Stereotactic body radiation therapy as an alternative to surgery in early-stage non-small-cell lung cancer. Oncology (Williston Park) 31: 492-498, 2017.

6. Wan Y, Yuan Y, Pan Y and Zhang Y: Antitumor activity of high-dose pulsatile gefitinib in non-small-cell lung cancer with acquired resistance to epidermal growth factor receptor tyrosine kinase inhibitors. Exp Ther Med 13: 3067-3074, 2017.

7. Zhang K and Yuan Q: Current mechanism of acquired resistance to epidermal growth factor receptor-tyrosine kinase inhibitors and updated therapy strategies in human nonsmall cell lung cancer. J Cancer Res Ther 12 (Suppl): C131-C137, 2016.

8. Pan L, Chai H and Kinghorn AD: The continuing search for antitumor agents from higher plants. Phytochem Lett 3: 1-8, 2010.

9. Shen P, Wang HG, Li MM, Ma QY, Zhou CW, Pan F and Xie R: Isofraxidin inhibited proliferation and induced apoptosis via blockage of Akt pathway in human colorectal cancer cells. Biomed Pharmacother 92: 78-85, 2017.

10. Niu X, Xing W, Li W, Fan T, Hu H and Li Y: Isofraxidin exhibited anti-inflammatory effects in vivo and inhibited TNF- $\alpha$ production in LPS-induced mouse peritoneal macrophages in vitro via the MAPK pathway. Int Immunopharmacol 14: 164-171, 2012.

11. Khan S, Riaz N, Afza N, Malik A, Aziz-ur-Rehman, Iqbal L and Lateef M: Antioxidant constituents from Cotoneaster racemiflora. J Asian Nat Prod Res 11: 44-48, 2009.

12. Deyama T, Nishibe S and Nakazawa Y: Constituents and pharmacological effects of Eucommia and Siberian ginseng. Acta Pharmacol Sin 22: 1057-1070, 2001.

13. Yamazaki T and Tokiwa T: Isofraxidin, a coumarin component from Acanthopanax senticosus, inhibits matrix metalloproteinase-7 expression and cell invasion of human hepatoma cells. Biol Pharm Bull 33: 1716-1722, 2010.

14. Qian L, Li X, Ye P, Wang G, Dai W, Liu Y, Gao Q and Shen G: Oxymatrine induces apoptosis and inhibits invasion in Gallbladder carcinoma via PTEN/PI3K/AKT pathway. Cytotechnology 70: 83-94, 2018.

15. Hu S, Ran Y, Chen W, Zhang Y and Xu Y: MicroRNA-326 inhibits cell proliferation and invasion, activating apoptosis in hepatocellular carcinoma by directly targeting LIM and SH3 protein 1. Oncol Rep 38: 1569-1578, 2017. 
16. Bai L, Lin G, Sun L, Liu Y, Huang X, Cao C, Guo Y and Xie C: Upregulation of SIRT6 predicts poor prognosis and promotes metastasis of non-small cell lung cancer via the ERK1/2/MMP9 pathway. Oncotarget 7: 40377-40386, 2016.

17. Wang Y, Wan D, Zhou R, Zhong W, Lu S and Chai Y: Geraniin inhibits migration and invasion of human osteosarcoma cancer cells through regulation of PI3K/Akt and ERK1/2 signaling pathways. Anticancer Drugs 28: 959-966, 2017.

18. Li W, Sun D, Lv Z, Wei Y, Zheng L, Zeng T and Zhao J: Insulin-like growth factor binding protein- 4 inhibits cell growth, migration and invasion, and downregulates COX-2 expression in A549 lung cancer cells. Cell Biol Int 41: 384-391, 2017.

19. Zhang L, Ge C, Zhao F, Zhang Y, Wang X, Yao M and Li J: NRBP2 overexpression increases the chemosensitivity of hepatocellular carcinoma cells via Akt signaling. Cancer Res 76: 7059-7071, 2016.

20. Niu X, Wang Y, Li W, Mu Q, Li H, Yao H and Zhang H: Protective effects of Isofraxidin against lipopolysaccharide-induced acute lung injury in mice. Int Immunopharmacol 24: 432-439, 2015.

21. Edlich F: BCL-2 proteins and apoptosis: Recent insights and unknowns. Biochem Biophys Res Commun: Jul 1, 2017 (Epub ahead of print).

22. Zhou L, Cai X, Han X, Xu N and Chang DC: CDK1 switches mitotic arrest to apoptosis by phosphorylating Bcl-2/Bax family proteins during treatment with microtubule interfering agents. Cell Biol Int 38: 737-746, 2014.

23. Spencer SL and Sorger PK: Measuring and modeling apoptosis in single cells. Cell 144: 926-939, 2011.

24. Hu X, Li D, Zhang W, Zhou J, Tang B and Li L: Matrix metalloproteinase-9 expression correlates with prognosis and involved in ovarian cancer cell invasion. Arch Gynecol Obstet 286: $1537-1543,2012$.

25. Kessenbrock K, Plaks V and Werb Z: Matrix metalloproteinases: Regulators of the tumor microenvironment. Cell 141: 52-67,2010.
26. Wang L, Wu H, Wang L, Zhang $\mathrm{H}$, Lu J, Liang Z and Liu T: Asporin promotes pancreatic cancer cell invasion and migration by regulating the epithelial-to-mesenchymal transition (EMT) through both autocrine and paracrine mechanisms. Cancer Lett 398: 24-36, 2017.

27. Yang L, Liu Y, Wang M, Qian Y, Dong X, Gu H, Wang H, Guo S and Hisamitsu T: Quercetin-induced apoptosis of HT-29 colon cancer cells via inhibition of the Akt-CSN6-Myc signaling axis. Mol Med Rep 14: 4559-4566, 2016.

28. Wang H, Chen B, Duan B, Zheng J and Wu X: miR-205 suppresses cell proliferation, invasion, and metastasis via regulation of the PTEN/AKT pathway in renal cell carcinoma. Mol Med Rep 14: 3343-3349, 2016

29. Chan LP, Liu C, Chiang FY, Wang LF, Lee KW, Chen WT, Kuo PL and Liang CH: IL-8 promotes inflammatory mediators and stimulates activation of p38 MAPK/ERK-NF- $\mathrm{BB}$ pathway and reduction of JNK in HNSCC. Oncotarget 8: 56375-56388, 2017.

30. Cepeda MA, Evered CL, Pelling JJH and Damjanovski S: Inhibition of MT1-MMP proteolytic function and ERK1/2 signalling influences cell migration and invasion through changes in MMP-2 and MMP-9 levels. J Cell Commun Signal 11: 167-179, 2017.

31. Gur S, Sikka SC, Abdel-Mageed AB, Abd Elmageed ZY, Rezk B, Pankey E, Kadowitz PJ and Hellstrom WJ: Imatinib mesylate (gleevec) induces human corpus cavernosum relaxation by inhibiting Receptor Tyrosine Kinases (RTKs): Identification of new RTK targets. Urology 82: 745.e11-6, 2013.

32. Puri N and Salgia R: Synergism of EGFR and c-Met pathways, cross-talk and inhibition, in non-small cell lung cancer. J Carcinog 7: 9, 2008.

33. Liu TC, Jin X, Wang Y and Wang K: Role of epidermal growth factor receptor in lung cancer and targeted therapies. Am J Cancer Res 7: 187-202, 2017. 